\title{
Designing Robots for Care: Care Centered Value-Sensitive Design
}

\author{
Aimee van Wynsberghe
}

Received: 29 April 2011/Accepted: 11 December 2011/Published online: 3 January 2012

(C) The Author(s) 2011. This article is published with open access at Springerlink.com

\begin{abstract}
The prospective robots in healthcare intended to be included within the conclave of the nurse-patient relationship_-what I refer to as care robots-require rigorous ethical reflection to ensure their design and introduction do not impede the promotion of values and the dignity of patients at such a vulnerable and sensitive time in their lives. The ethical evaluation of care robots requires insight into the values at stake in the healthcare tradition. What's more, given the stage of their development and lack of standards provided by the International Organization for Standardization to guide their development, ethics ought to be included into the design process of such robots. The manner in which this may be accomplished, as presented here, uses the blueprint of the Value-sensitive design approach as a means for creating a framework tailored to care contexts. Using care values as the foundational values to be integrated into a technology and using the elements in care, from the care ethics perspective, as the normative criteria, the resulting approach may be referred to as care centered valuesensitive design. The framework proposed here allows for the ethical evaluation of care robots both retrospectively and prospectively. By evaluating care robots in this way, we may ultimately ask what kind of care we, as a society, want to provide in the future.
\end{abstract}

Keywords Value-sensitive design - Care robots · Care ethics ·

Ethics and technology

\section{Introduction}

As roboticist Joseph Engelberger predicted in 1989, the use of robots no longer remains exclusively in the domain of the factory (1989). One of the most intriguing

\footnotetext{
A. van Wynsberghe $(\bowtie)$

Department of Philosophy, Faculty of Behavioral Sciences, University of Twente,

P.O. Box 217, 7500 AE Enschede, The Netherlands

e-mail: aimeevanwynsberghe@hotmail.com
} 
and morally challenging applications of robots is their use in healthcare scenarios. The most prominent and widespread are surgical robots, the daVinci ${ }^{\mathrm{TM}}$ Surgical System or its predecessor the Zeus ${ }^{\mathrm{TM}}$ Telesurgical System for example (Van Wynsberghe and Gastmans 2008); however, the more recent and intriguing robots to be used in healthcare are those intended for inclusion in the daily care activities of persons, activities like lifting, feeding or bathing. These robots, what I refer to as care robots, will be used by the care-giver in the care of another or may also be used by the care-receiver directly. The initiative to create such robots stems from the foreseen lack of resources and healthcare personnel to provide a high standard of care in the near future: fewer people in the younger generations are available to meet the care needs of the ageing population (WHO 2010). This trend is expected to be observed in multiple countries. Consequently, the idea is that this wave of automation, namely care robots, may help to mitigate the coming lack of care workers by providing assistance during care tasks or by fulfilling care tasks to relieve time for the many duties of care workers. Given the sensitive and morally complex context(s) into which these robots will be stepping, ethical attention to their potential impact is called for. To date, authors like Sharkey and Sharkey (2010) and Shannon Vallor (2011) have addressed certain issues pertaining to the use of care robots by addressing their potential impact on the rights of elderly citizens or the development of care-givers in their personal and professional roles, respectively.

In line with such ethical evaluations, attempts have been made to outline a framework for the ethical evaluation of robots (Veruggio and Operto 2006), or to suggest the dimensions of a robot ethic (Asaro 2006). These efforts, however, fall short by neglecting certain technical aspects, neglecting the specific issues pertaining to care contexts (for robots in healthcare), or failing to provide the structure of a framework at all. A framework for the ethical evaluation of care robots requires recognition of the specific context of use, the unique needs of users, the tasks for which the robot will be used, as well as the technical capabilities of the robot. Above and beyond a retrospective evaluation of robots, however, what is needed is a framework to be used as a tool in the design process of future care robots to ensure the inclusion of ethics in this process. What's more, given the lack of standards provided by the International Organization for Standardization (ISO 2011), there exists an opportunity at this time to incorporate ethics into the actual design processes for these kinds of robots. Accordingly, if ethics is to be included in the design process of robots, one must first identify the moral precepts of significance followed by an account as to how to operationalize said precepts.

Accordingly, this work addresses the following question: how can care robots be designed in a way that supports and promotes the fundamental values in care. As such, I neither refute the potential positive contributions of care robots nor the negative. Rather, I am looking for the most desirable way in which to proceed with their design. The following paper begins by outlining what a care robot is and why questions of design are the most significant questions to address at this time for these robots. Following this, I embark on a conceptual investigation of the concepts of value, and of care, for understanding their complexity. From this, I present a normative framework for the retrospective and prospective evaluation of care robots and provide an example to illustrate its utility. 


\section{What is a Care Robot?}

As I have mentioned, a care robot is one that is used in the care of persons in general. For Vallor, "carebots are robots designed for use in home, hospital, or other settings to assist in, support, or provide care for the sick, disabled, young, elderly or otherwise vulnerable persons" (2011). According to Sharkey and Sharkey, the tasks for which the care robot is used can be classified in terms of either providing assistance in caregiving tasks, monitoring a patient's health status and/or providing social care or companionship (2010). Both Vallor and Sharkey and Sharkey, list examples of current care robot prototypes from the RI-MAN autonomous lifting robot at the Riken Institute, now replaced with the RIBA, to the MySpoon feeding assist robot. Care robots are also envisioned for tasks like bathing as well as social companionship, the most well known of the latter being the Paro robot. Most recently, the car company Toyota has announced the release of four care robots intended to aid a nurse with lifting patients or to assist patients with walking. ${ }^{1}$

As we can see, there is no capability exclusive to all care robots rather; they may have any number and range of capabilities from planar locomotion (vs. stationary) to voice recognition, facial or emotion recognition. Additionally, they may have any degree of autonomy, from human-operated (as in the surgical robot daVinci) or varying degrees of autonomy (like the TUG robot for deliveries in the hospital which requires minimal human input or the RIBA robot intended for lifting patients without input from a human user). Thus, the definition of a care robot relies on the idea of interpretive flexibility, that a robot is defined by its context, users and task for use (Howcroft et al. 2004). This means that the same robot might be called by a different name if the robot is used for rehabilitation or for care purposes. The Hybrid Assistive Limb (HAL) is an example of this phenomena; the robot may be used in rehabilitation when worn by a patient (Kawamoto and Sankai 2002) or could be used to relieve the stress of lifting on the nurse. For the purposes of this work, a care robot will be defined as such according to its application domain (hospital, nursing home, home setting), its intended use (a care practice deemed as such according to its use domain) and its intended users (care givers and/or care receivers, in a care domain for a care practice.

\section{Creating a Framework for the Ethical Evaluation of Robots}

When discussing ethics and robots, current authors approach the question from a variety of perspectives; the rights of individuals (Sharkey and Sharkey 2010), the specific needs of a care receiving demographic (Sparrow and Sparrow 2006), or the impact specific to the care-giver (Vallor 2011). While these analyses provide useful contributions to the question of ethics and care robots at large they lack the formation of normative recommendations based on their work for the design of future care robots. In Peter Asaro's article "What should we want from a robot ethic?" (2006), he proposes the three dimensions one could be referring to when one

\footnotetext{
${ }^{1}$ http://news.cnet.com/8301-17938_105-20128993-1/toyota-plans-nursing-robots-for-aging-japan/.
} 
says "ethics of robots": (1) the ethical systems built into robots; (2) the ethical systems of people who design robots, and; (3) the ethics of how people treat robots. He then concludes that given the nature of robots as socio-technical systems, a framework for ethically addressing robots ought to include all three dimensions. For Asaro, the overarching question that each of the three dimensions stem from has to do with the distribution of moral responsibility in the social-technical network into which robots are introduced. Asaro presents a compelling case for the need for a comprehensive approach to robot ethics, but stops short of presenting such an approach. In this paper, I intend to take up this challenge. To that end I have created a framework which incorporates ethical analysis, according to the care perspective, into the design process of a care robot. The goal with this framework is threefold; to stimulate ethical reflection of designers/engineers, to encourage ethical reflection from the care ethics tradition, and to illuminate the relationship between the technical content of a care robot and the resulting expression of care values within a care practice.

To accomplish these goals, I argue that the approach known as value-sensitive design (VSD) adequately addresses the three dimensions identified by Asaro as well as his overarching question. Value-sensitive design is defined as "a theoretically grounded approach to the design of technology that accounts for human values in a principled and comprehensive manner throughout the design process" (Friedman and Kahn 2003). Value-sensitive design takes as its starting point the belief that technologies embody values (the embedded values approach) and offers a coherent method for evaluating the current design of technologies but also offers a proactive element to influence the design of technologies early on and throughout the design and implementation process. This concept refutes the neutrality thesis of computer systems and software programs which states that such systems are in themselves neutral and depend on the user for acquiring moral status. Instead, it is possible to identify tendencies within a computer system or software that promote or demote particular moral values and norms (Brey 2009; Nissenbaum 1998). These tendencies manifest themselves through the consequences of using the object. When said technology is capable of imposing a behavior on a user, or consequence to using it, the imposing force within the technology is considered a "built-in" or "embedded" value (or alternatively a disvalue if the computer system hinders the promotion of a value). The consequence is considered a special kind of consequence to using the object; one that brings about the promotion or demotion of a cultural value (Brey 2009). To give a simple example of this phenomenon, one may think of a personal banking machine-the ATM, through its use, promotes a certain value of user autonomy or distributive justice. At the same time, the ATM machine enforces certain biases of users - that they are a certain height and are literate in order to use the machine. Observing values within a system is a complex endeavor whereby the promotion of one value may be fulfilled while at the same time there is a trade-off with another value.

Accordingly, technologies may be designed in a way that accounts for values of ethical importance in a systematic way and rigorously works to promote said values through the architecture and/or capabilities of a technology. It follows then that care robots may be designed in a way that promotes the fundamental values in care. 
Although VSD is meant for the design of a particular system or product, that is not my overall aim. My goal is to create a general framework that may be used by designers and/or ethicists in the ethical evaluation of any care robot, or for the inclusion of ethics in the design of any care robot. By using the blueprint of VSD I am creating a framework that addresses the specific relationship between technical capabilities and design of care robots with the specific context of use, task of use and users in mind. An additional benefit to the framework is its potential for use retrospectively and prospectively. When used retrospectively, designers are able to understand the impact of their design on the resulting care practice. When used prospectively, designers are able to incorporate the framework into the design process of a care robot, ultimately incorporating ethics into the design process.

Value-sensitive Design has been praised by computer ethicists and designers for its success in incorporating ethics in the overall design process of computer systems or ICT (Van den hoven 2007) but is also advantageous to guide the design process of a wide array of technologies (Cummings 2006). The framework I am creating uses components of the VSD methodology in its creation-namely the conceptual investigation coupled with a brief empirical and technical investigation. As in traditional VSD, my conceptual investigation is an exploration of the value constructs of the values of ethical importance, for this work the values of ethical importance are those from the care ethics tradition. VSD, however, has also been criticized for its lack of normative grounding given that it rests on rather abstract values without an ethical theory to anchor their interpretation (Mander-Huits 2011). With this in mind, I diverge from traditional VSD in that I utilize the findings from the care ethics perspective to guide the discussion of relevant care values and their meaning as well as the manner in which the ethical analysis ought to take place. Based on the work of care ethicist Joan Tronto, I claim the fundamental care values of any practice to be attentiveness, responsibility, competence and reciprocity. I attempt to understand how these values are interpreted philosophically by care ethicists, as well as how these values are interpreted in context through observational work. The resulting framework thereby provides a normative account of the values in care. Putting the framework to use incorporates a technical investigation by exploring technical capabilities of care robots currently available or available in the near future. This highlights the relationship between technical content and the resulting expression of values. Unlike the traditional empirical and technical components of VSD, I do not embark on empirical studies to test a care robot in context with human users (at least not at this moment in time). This is because I aim to provide a framework for the design of a range of care robots and not one particular system. In order to utilize the framework for evaluation it is necessary to shift back and forth from conceptual to empirical to technical aspects, in much the same manner as other VSD methodologies.

\section{Why Begin with Design?}

Not only do the three dimensions presented by Asaro point towards a discussion of design but the answer to the question of why one ought to pay so much attention to 
issues of design is further grounded in three rationales. Firstly, there are no (universal) guidelines or standards for the design of robots outside the factory. Although the International Organization for Standardization has currently drafted standards for design of personal robots, these are classified differently from medical-use robots. Care robots will most often be found in medical settings like a hospital or nursing home and no such standards for medical robots exist to date. As a result, designers are given no guidelines pertaining to the inclusion of socially sanctioned ethical principles like safety and/or efficiency, principles which designers still strive for but do so without any standardized means. Secondly, the nascent stage of the development of robots. Given the multi-disciplinary nature of robotics a range of disciplines are involved in the design of a system. Disciplines range from computer science to engineering and from sociology to psychology. This does not mean there is neither a need nor an interest to incorporate ethics in the design process but rather there has been no attempt to facilitate a way in which ethics may be translated for engineers/designers.

The necessity for inclusion of ethical criteria throughout the design process of a care robot brings us to the third rationale-that of the far reaching impact technologies have on societies (good or bad) and in turn, that societal values and norms have on the development of a technology. From the perspective of the philosophy of technology, many theories exist which seek to explain the reciprocal and dynamic relationship between society and the development of technologies. This may not be an explicit aim of the designer but is a condition of the work that they do. The theory of scripts illustrates how engineer's assumptions about user preferences and competencies show themselves in the technical content of an object (Akrich 1992). Latour expands this idea to show how technologies steer behaviors, moral and otherwise (1992). Verbeek shows how technologies are included in our decision making such that moral decisions are in fact a hybrid affair between humans and technologies (2006). In the computer ethics domain, Nissenbaum illustrates how values and biases are embedded into a computer system (1998). The embedding of values and of biases and the intertwining of the two was seen previously in the ATM example. The golden thread through all of these perspectives is that social norms, values and morals find their way into technologies both implicitly and explicitly and act to reinforce beliefs or to alter beliefs and practices.

Beyond the embedding of values and/or norms, once the robot enters a network it will alter the distribution of responsibilities and roles within the network as well as the manner in which the practice takes place. This shift is what Verbeek refers to as mediation: "when technologies are used, they help to shape the context in which they fulfill their function, they help to shape human actions and perceptions, and create new practices and ways of living" (Verbeek 2008, p. 92). Akrich discusses this in terms of the assumptions designers have of the traditional and ideal distribution of roles and responsibilities - that practices may shift based on an assumption made by an engineer of how the practice "ought" to take place, how roles and responsibilities "ought" to be delegated, and inscribing these assumptions into the technical content. For Akrich, "many of the choices made by designers can be seen as decisions about what should be delegated to a machine and what should be left to the initiative of human actors" (p. 216). By making choices about what should and should not be 
delegated to certain actors (human or nonhuman), engineers may change the distribution of responsibilities in a network. Or as Verbeek claims, engineers are 'materializing morality' (2006). It is these ideas that mirror the overarching question presented by Asaro - that a robot ethic ought to address the shift in responsibilities once the robot has been included into a socio-technical network. What's more, when a shift in roles and/or responsibilities is inscribed in a robot a valuation is being made-for example, that the human is not competent to fulfill the task or that the robot may fulfill the task in a superior manner. Thus, even assumptions about users may be considered statements of value, or normative claims, at times.

It is true that the rationales presented here relate to the design of any system or technology; however, greater weight is added when one takes into account the context in which the care robot will be placed and the nature of the activities the care robot will fulfill. Meaning, without standards guiding the development of care robots how is one to be sure that the values and norms central to the healthcare tradition will be promoted? Or, without making these norms and values explicit through the design process, how can one be sure their inclusion will be taken into account? Or, given the cost of development of these robots, mustn't they provide the same quality of care as today if not better (which presupposes an understanding of how one defines "good care")? Or simply, given the dramatic impact care robots may have on society, shouldn't future considerations be taken into account in design? With these rationales in mind, the design of any technology is ultimately a moral endeavor. The design of a care robot then is even more so given the vulnerability of this demographic, the delicacy of their care needs and the complexity of care tasks.

\section{Exploring the Concepts of 'Value' and of 'Care'}

To begin the creation of the framework, I embark on a conceptual investigation of the concepts of 'value' and of 'care'. By uncovering the values of ethical importance in a care context, the aim is to expose the moral precepts to operationalize in the design of a care robot.

\section{Defining Values}

According to the Oxford English Dictionary, values are conceived of as "the principles or standards of a person or society, the personal or societal judgment of what is valuable and important in life" (Simpson and Weiner 1989). Thus, a value is something desirable, something we want to have or to have happen. It follows then that when something is de-valued it loses importance. Values may be intrinsic or inherent to an object, activity or concept, or, things may be valued as a means to an end (Rosati 2009). For example, in the healthcare context, the concept of human dignity is valued on its own whereas the activity of touch in care contexts is valued as a means to preserving the dignity of persons (Gadow 1985). Things of value ${ }^{2}$ may

\footnotetext{
${ }^{2}$ I have used the word 'things' here to bypass repeating people, places, activities, concepts, and objects, all of which are included in the discussion of values.
} 
be valued on a personal level or on a societal/cultural level. Values then may be more of a subjective enterprise (various things valued for an individual) or more of an objective enterprise (universal values such as justice, human dignity, fairness). The latter does not imply that values considered abstract and universal are interpreted in the same way between cultures or time periods but rather that the valuation of things may differ from an individual's sphere to a more public one. Linked with the concept of 'good', a value may be construed as something that is good or brings about a good consequence.

In the VSD literature, Batya Friedman and colleagues, opt for a more open definition of a value to refer to "what a person or group of people consider important in life" (Friedman and Kahn 2003, p. 2). This implies then that all the values are not interpreted in the same way. Nathan et al. illustrate this with the value of privacy and its divergent ways of being interpreted between cultures and therefore protected (2008). Le Dantec et al. reinforce the idea that values may be universal, or generally accepted, but differ in their interpretation. Because of this, Le Dantec et al. suggest a way in which the methodology of VSD may be strengthened, through an uncovering of values in situ or discovering values through experiencing the practice (Le Dantec et al. 2009). This is of course due to the idea that differences exist between designers' values and users' values (Nathan et al. 2008). Thus, the scope of values varies depending on the technology, the users, the culture, the time period and the application domain. In the VSD methodology, Friedman selects the values of ethical importance pertaining to computer systems. Given that my framework is intended for use in the design of care robots, the values pertaining to the specific context are of greater ethical significance and relevance.

\section{Defining Care, Care Ethics and Care Values}

Care may be one of the most difficult concepts to articulate. This is in part due to the ubiquity of the word but is also largely a consequence of the fact that one is assumed to know what care means given its revered place in many cultures. The work of Warren T. Reich nicely outlines the broad range of meanings and connotations care has embodied going back as early as Ancient Greece (Reich 1995). Regardless, of how one perceives or defines care, care is valued as something above and beyond simple care giving tasks. It has a central role in the history of human kind as a means to signify the value of others. In other words, by caring you bestow value on the care-receiver.

In the verb "to care" one finds that caring may actually be divided into the idea of caring about and caring for. The dimension of caring about in the medical field implies a mental capacity or a subjective state of concern. On the other hand, caring for implies an activity for safeguarding the interests of the patient. In other words, it is a distinction between an attitude, feeling or state of mind versus the exercise of a skill with or without a particular attitude or feeling toward the object upon which this skill is exercised (Jecker et al. 2002). In the field of care ethics, Joan Tronto claims that good care is the result of both a caring attitude in combination with a caring activity (Tronto 1993). In other words, a marriage between the dimensions of caring about and caring for. 
The field of care ethics is most often attributed to the Kohlberg-Gilligan debate on moral psychology (Gilligan 1982). Because of this debate, a new way of perceiving the moral dilemma in a given scenario arose; one that shifted the central focus from rights and universally applicable rules to a focus on responsibilities and relationships as central factors. Perhaps the most significant result that came from Gilligan's work (along with the assistance of other scholars) is the understanding of care ethics as a perspective or orientation from which one begins to theorize rather than a pre-packaged ethical theory. In fact, care ethicists are not striving to arrive at some ready-made theory for application (as in traditional ethical theories) but rather point towards the necessary beliefs or elements that structure the care orientation. These beliefs refer to:

an emphasis of concern and discernment (to notice and worry more about the dangers of interference rather than the dangers of abandonment), habits and proclivities of interpretation (the proclivity to read the moral question presented by a situation in terms of responsibilities rather than rights), and selectivity of skills (to have developed an ease of abstraction more than an attunement to difference) (Little 1998, p. 195).

As Little articulates, "the orientations provide illuminating stances from which to develop ethics of these relationships, not that they constitute those ethics readymade" (Little 1998, p. 206). This is precisely what my aim is when developing the framework for the ethical analysis and evaluation of care robots-to outline an orientation from which the ethical evaluation may begin by emphasizing certain fundamental components in care.

Aside from a conversation about the concept of care or the care ethics perspective, there is much to say about care values. Alternative to the idea that care in itself is a value-linked with the good life and with a valuation of another-is the idea that beneath the umbrella concept of care comes many other values. These values are given importance for their role in care-their role in giving significance to care, in making care what it is. These values form the buttress for care as an ethical endeavor and create a framework for evaluating care as a practice. It is through the manifestation of these values that one comes to understand what care really is in practice. It is therefore fruitful for the topic of embedding care values, to understand these values and their link with consequences. Thus, to begin from a topdown approach, I look to the values articulated by the governing body of healthcare, namely the World Health Organization (WHO). The WHO framework for peoplecentered health narrows in on the values in healthcare stemming from the patient's perspective; patient safety, patient satisfaction, responsiveness to care, human dignity, physical wellbeing and psychological wellbeing (2007). This is not to say that other values like innovation or physician autonomy are not valued but rather from the patient's perspective, the listed values are the ones with the greatest ethical importance and will thus be used in my evaluation of implementing robots in the care of persons.

Without an understanding of the specific context or the individual characteristics of a patient, these values don't tell the engineer much concerning how the value may be embedded in a care robot prototype. Therefore, I take the suggestions of 
Le Dantec et al. to understand the specific interpretation of these values in context, achieved through fieldwork experience in both a hospital and a nursing home. ${ }^{3}$ Interestingly, the interpretation of values as well as their ranking and meaning differed depending on: the type of care (i.e. social vs. physical care), the task (ex. bathing vs. lifting vs. socializing), the care-giver and their style, as well as the care-receiver and their specific needs. For example, in a ward with people suffering from dementia, safety is in terms of not letting patients wonder onto the streets, or preventing patients from hurting both themselves and others. In a 'typical' ward of a nursing home, safety is in terms of preventing patients from falling, or assisting in the feeding of patients to prevent chocking. How a value is prioritized is also dependent on the context, personal experiences but also the specific practice. For example, through the practice of lifting, the value of safety is manifest (or interpreted) by ensuring the care-receiver does not fall or is not injured. Here, safety is of paramount importance. In contrast, through the practice of bathing, the value of safety is interpreted in terms of suitable water temperature (not burning or scarring the patient), and proper positioning on the bed or tub to prevent injury. In the practice of bathing, however, while safety is of the utmost importance, other values take precedence. For example, closing the curtain to ensure privacy, verbal communication to calm the care-receiver, and gentle strokes to convey empathy and respect through the practice. These examples make us aware of both the intertwining of care values and the actions of care-givers but also the significance of the therapeutic relationship-all of the values central to the healthcare tradition are observable within the relationship, the actions and interactions between the nurse and the patient.

When wondering about the relationship between technologies and care values, the value of touch helps to shed light. Touch is an important action in care that is valued on its own as well as a means for manifesting other values like respect, trust and intimacy. Touch is the symbol of vulnerability, which invokes bonds and subjectivity (Gadow 1985). Touch acts to mitigate the temptation for objectification. Thus, touch is considered an instrumental value in the healthcare domain, the outcome of which results in the preservation of the value of human dignity. Using the value of touch as an example, we can see how a certain technology might impede its manifestation. Melanie Wilson illustrated how a particular computer system implemented in the field of nursing was rejected as it prevented nurses from "hands on care" - from touch - a cornerstone of the nursing tradition (Wilson 2002). One might suggest that designers of this technology were not aware of the significance of 'hands-on' care for nurses even when the nurse's role is to create a daily care plan.

In short, not only is care a value for what it symbolizes (a valuation of another) and manifests (meeting the needs of another) but it is also valued for the additional elements that make up care; patient safety, patient satisfaction, responsiveness to care, human dignity, physical wellbeing and psychological wellbeing. The list of

\footnotetext{
${ }^{3}$ Fieldwork experience was gained by volunteering in a nursing home in London, Ontario, Canada for 4 weeks as a "life enrichment coach" as well as observing practices in multiple hospital, also in London, Canada.
} 
care values is exhaustive when one considers the significance of the therapeutic relationship and the elements within this conclave (trust, respect, compassion, empathy and touch); however, what is hopefully now evident is the significance of the care relationship in terms of the intertwining of care actions with care values. Meaning, the expression of care values are the result of the actions and interactions between actors. To explain this further, I turn to the concept of a care practice.

\section{Care Practices}

To elaborate on the marriage between caring about and caring for, a useful concept is that of a care practice. A care practice is, as care ethicist Joan Tronto describes it, a way to envision a care task or a series of care tasks. A way in which one can grasp the fortitude of each action and interaction between a care-giver and a care-receiver. More importantly, it is a way to envision the holistic nature of care.

The notion of a care practice is complex; it is an alternative to conceiving of care as a principle or as an emotion. To call care a practice implies that it involves both thought and action, that thought and action are interrelated, and that they are directed toward some end (Tronto 1993, p. 108).

Understanding that care tasks are more than just 'tasks' but are rich practices in a value-laden milieu that act to bring about the promotion of values, may be one of the most crucial points for designers to grasp. The reason for this has to do with understanding how values are manifest and thus how a design will impact this materialization. To exemplify this shift from task to practice, let me use the practice of lifting. When a patient is lifted by the care-giver, it is a moment in which the patient is at one of their most vulnerable. The patient trusts the care-giver and through this action a bond is formed and/or strengthened which reinforces the relationship between care-giver and the care-receiver. The significance of this is apparent in the actual practice of lifting but comes into play later on in the care process as well. Meaning trust, bonds, and the relationship, are integral components for ensuring that the care-receiver will comply with their treatment plan, will take their medication and be honest about their symptoms. Without trust, these needs of the care-giver are threatened, ultimately threatening the entire care process and the good care of the care-receiver. Thus, conceptualizing care tasks as practices adds a deeper meaning to each 'task'. It is within a care practice that the values are manifest and given their significance but it is also within practices that the holistic vision of care takes form-each care practice builds from, and on to, another practice linking all practices in the overall care process.

\section{Selecting the Values of Ethical Importance in Care}

While many care ethicists make clear the range of values and principles that provide a normative account for care (Vanlaere and Gastmans 2011; Little 1998; Ruddick 1995; Noddings 1984) they fall short of providing a systematic way to visualize and evaluate these principles and values. The vision presented by Joan Tronto allows for a perception of care as a process with stages and corresponding normative moral 
elements, which provides the most enticing conceptualization for engineers to work with. There are four phases of a care practice for Tronto; caring about (recognizing one is in need and what those needs are), care taking (taking responsibility for the meeting of said needs), care giving (fulfilling an action to meet the needs of an individual), care receiving (recognition of a change in function of the individual in need). These phases have corresponding moral elements as standards to evaluate the care practice from a moral standpoint. These elements are: attentiveness, responsibility, competence, and responsiveness. Attentiveness refers to an attribute or virtue of the care-giver, a certain competence for recognizing needs. Responsibility refers again to an element of the care-giver and their stance or concern for ensuring the care-receiver is pointed in the right direction for care or maintaining an accurate assessment of needs etc. Responsibility is often delegated to a moral agent; however, some responsibilities are delegated to an artifact as technologies are wide spread in healthcare. Here, the concept of mediation (Verbeek 2006) becomes critical in the sense that decision making on the part of nurses and patients is a hybrid affair between the nurse/patient and existing technologies. Competence is once again an attribute of the care-giver and refers to the skills with which the care is given. An unskilled care-giver may be more detrimental than no care at all. Responsiveness refers to an attribute of the care-receiver and their role in the relationship - to guide the care-giver. This element (and the phase of care receiving) is important for remembering the reasons for care in the first place: the care-receiver and their needs. Without this, care is not complete. This recognition also encourages an active stance of the care-receiver rather than a more passive, vulnerable one.

Creating a standardized framework to guide the promotion of these values which applies to any care context, task, care-receiver or care-giver reveals itself to be quite problematic given the range and variety of care values discussed in the former section. In other words, to claim that human dignity, compassion or respect for power are values to be embedded in a care robot offers nothing for the designer in terms of the robot's capabilities. Moreover, as we have seen, their ranking and prioritization is dependent on the context (i.e. one hospital domain or another vs. a nursing home) and task (ex. lifting vs. bathing). To standardize the creation of care robots there needs to be another avenue besides values alone. In the care ethics literature, alongside values, need too play a central and crucial role in the provision of good care. The needs of the patient mark the starting point of the care process and the process then revolves around a care-giver (or multiple care-givers) taking steps to meet these needs. Understanding the multiple layers of needs, the many ways in which they might be fulfilled, the preferences for one way over another, and the divergent needs between individuals, adds a further complexity to the meeting of needs. If this wasn't complicated enough, the care-giver has needs too! Needs in terms of resources, skills, responsiveness from the care-receiver to understand when needs have been met as well as their own personal needs.

Given the central role of needs in a care context, what might the relationship be between needs and values? Although many authors have written on the subject, little consensus can be found. I suggest that the values in healthcare are given their importance for their role in meeting needs. This corresponds with Super's conceptualization of the relationship between needs and values: "values are 
objectives that one seeks to attain to satisfy a need" (1973, pp. 189-190). Meaning, the value is the goal one strives towards and in so doing, intentionally meets a need. Thus, we begin with needs, and the values represent the abstract ideals which, when manifest, account for the needs of individuals. It follows then that a framework for designing care robots ought to address the meeting of needs. But not so fast, we've just shown how multifaceted and intricate needs are for the care-giver and carereceiver. What's more, according to the field of care ethics, it is neither possible nor advisable to outline a series of needs which pertain to all care-givers, care-receivers or care tasks in every instance/scenario (Tronto 2010). While useful for policy or a universal ethical code, it goes against the vital element in care-that of the individual and their unique, dynamic needs. In other words, care is only thought of as good care when it is personalized (Tronto 1993). There is, however, a solution to this barrier. It is possible to delineate a set of needs for every care practice. To recapitulate, together the phases and the moral elements make up a care practice. The practices are values working together and the vehicle for this is the moral elements. If we assume a care practice ought to proceed according to Tronto's phases than the needs for every care practice are the corresponding moral elements. It is therefore these elements that ensure the promotion of care values. Consequently, it is these elements-attentiveness, responsibility, competence, responsiveness - that make up the normative portion of the framework.

With this suggestion, there are two assumptions being made; that every care practice will always have the moral elements as needs, independent of the care-giver and care-receiver, and that the values are subsumed within the moral elements. Using the practice of feeding as an example to illustrate the first assumption, I am making the claim that this practice will always require attentiveness, responsibility and competence on the part of the care-giver and will always require a reciprocal interaction between care-receiver and care-giver for determining whether or not the needs have been met, no matter who the care-giver is or who the care-receiver is. In other words, these moral elements are independent of the actors. They are, however, dependent on the context and the specific practice for their interpretation and prioritization. If we were to compare the practice of lifting with the practice of feeding we would see how the element of competence is uniquely interpreted in each practice (skillfully bearing the weight of another without dropping or causing pain vs. skillfully coordinating timing and placement of food and utensils). In terms of context, the practice of lifting in the hospital requires greater efficiency than the practice of lifting in a home setting where time may not be as much of an issue. Thus, although the moral elements must always be present, the context and practice still play a crucial role in their interpretation, prioritization and manner of manifestation.

For the second assumption-that the values are subsumed within the moral elements-one may find that the values are often analogous to a phase or moral element or are expressed through the manner in which an action takes place. The value of patient safety is fulfilled through the competent completion of a practice (the phase being care giving and the moral element being competence). The valued action of touch requires attentiveness on the part of the care-giver for determining when and to what degree touch is considered necessary. The manner in which care 
practices take place is often tailored to the specific likes of one care-giver or another and again requires attentiveness to those preferences and competence in meeting them. What's more, paying attention to those unique preferences is a vehicle for establishing trust and allowing for successful reciprocal interaction.

In short, ensuring that the elements are present or strengthened through the design and introduction of a care robot, ultimately results in a manifestation of the core care values. Differences in the prioritization and manifestation of moral elements between practices and/or contexts is something that the care ethicist may draw the attention of the designer to while utilizing the framework throughout the design process. Nevertheless, the designer must first be aware of the necessary elements and their manner of manifestation.

\section{The Care-Centered Framework}

By summarizing and synthesizing the findings thus far I arrive at a framework to be used in the design process of care robots, both by ethicists as well as engineers/ designers. The framework for the design of care robots is distinguished from the method to proceed when using the framework. The latter is referred to as the "care centered value sensitive design" (CCVSD) methodology and the former is referred to as the "care centered framework". The care centered framework and CCVSD methodology both pay tribute to the central thesis in care ethics, namely that the care perspective provides an orientation from which to begin theorizing as opposed to a pre-packaged ethical theory. The framework articulates the components that require attention for analysis from a care perspective while the methodology indicates how these components are to be analyzed with and without the introduction of a care robot. The care centered framework aims to outline the orientation from which one begins in order to develop an ethic of the relationship between care robot and the other actors involved in the care practice. The framework consists of five components: context, practice, actors involved, type of robot, and manifestation of moral elements (see Table 1). Each of these components will be described in detail for understanding their place within the framework from the care ethics stance.

The framework is intended to be a general outline for the creation of any care robot and not one care robot in particular for one practice in one context. Thus, the

Table 1 Framework for the ethical evaluation of care robots

\footnotetext{
Context-hospital (and ward) versus nursing home versus home...

Practice-lifting, bathing, feeding, delivery of food and/or sheets, social interaction, playing games...

Actors involved - nurse and patient and robot versus patient and robot versus nurse and robot...

Type of robot-assistive versus enabling versus replacement...

Manifestation of moral elements-Attentiveness, responsibility, competence, responsiveness
}

The ellipsis following the description of a criterion indicates that the list is not exhaustive and may include additions 
framework is standardized with respect to designing care robots in general according to the necessary components but is not standardized with respect to dictating how each value is interpreted and ranked for a care practice. This is done on a case-by-case basis for each practice in a given context. This is so due to the difference in capabilities of the robot depending on the practice and context for which the robot is intended. Meaning, a robot designed for delivery of sheets will have distinctly different capabilities from a robot designed for feeding.

\section{Context as a Component of the Framework}

Firstly, one must identify the context within which the care practice is taking place. For example, the specific hospital and the ward versus a nursing home versus a home setting. The context within which the care practice takes place is important for a variety of reasons. Recent research indicates a relationship between religious beliefs and one's acceptance of using robots in care-taking roles (Metzler and Lewis 2008). Metzler and Lewis are investigating the hypothesis that when one believes in "a god" they may not be as inclined to accept human-robot interaction with life-like robots at an intimate level. Thus, the design of a robot for a Catholic hospital ought to take this kind of research into consideration for the appearance of the robot. Similarly, the context in terms of one hospital ward or another is also of great importance when designing the robot. Research done by Bilge Mutlu of the University of Wisconsin, Madison (Barras 2009) shows how the same robot (the TUG robot) used in one hospital was accepted differently depending on the ward. Workers in the post-natal ward loved the robot, while workers in the oncology ward found the robot to be rude, socially inappropriate and annoying. The same workers even kicked the robot when they reached maximum frustration.

Specifying context in terms of a nursing home versus a home setting is also of importance given that the prioritization of values differs. For example, lifting in the nursing home places efficiency as a higher priority (and even more so in the hospital) while in the home setting there may not be the same time constraints. In addition, bathing in a home setting may not require the same demand for privacy as in the hospital or nursing home setting given the lack of other patients around. Specifying the context plays a crucial role for understanding the prioritization of values.

\section{Practice as a Component of the Framework}

The practice for which the care robot will be used plays a dominant role in the prioritization as well as the interpretation of values/moral elements. Examples of practices are lifting, bathing, feeding, fetching items, delivery of medications/food/ $\mathrm{x}$-rays/sheets to the room or to the nurse, personal communication, social interaction, games and activities like singing songs or painting. As mentioned, each of these practices requires the elements of attentiveness, responsibility, competence and reciprocity; however, they mean very different things depending on the type of practice. Competence in terms of lifting refers to a skilled lifter that does so at the appropriate speed and angle without hurting or dropping the care-receiver. Attentiveness to whether or not the care-receiver is being hurt or pinched in any way 
is also an attribute of competence. Alternatively, competence for the practice of feeding refers to the feeder gently, with great precision and at an appropriate speed bringing the utensils to the care-receiver's mouth. Thus, the interpretation of the elements are determined according to the practice for which the robot will be used.

\section{Actors Involved as a Component of the Framework}

From the care orientation, the actors involved are of great significance for structuring moral deliberation. One of the most important findings to come from the care ethics perspective is the ontological status of humans as relational. Its significance for this work lies in recognizing that the care practice which a robot will enter involves a network of human (and nonhuman) actors in relationship. The robot then has the potential to shift the roles and responsibilities distributed within these relationships. Alternatively, if the patient is receiving care in their home perhaps the actors involved are family members or a visiting nurse who is not present on a daily basis. Then again, a patient may fulfill certain practices on their own prior to a robot assisting. This does not mean the care-receiver is entirely on their on, in the atomistic sense, but rather that the robot may be delegated a certain portion of the role of the care-receiver (as is the case with a feeding robot like Secom's MySpoon). This component is meant to highlight the roles and responsibilities attributed to actors prior to the robot entering the scene. Throughout the evaluation (both retrospective and prospective), the goal is to understand how the traditional roles attributed to actors shift or remain the same.

It is important to remember too that the human actors are not acting alone to manifest values. They work together with each other but also with technologies already in use in the healthcare system. In nursing and technology studies, technologies have often been considered extensions of the nurse's body or self. Nurses become so skilled at using the technology they do so without being interfered by the technology's presence. What's more is that the nurse's role is one that incorporates the use of technologies in a variety of ways from the mechanical bed to heart monitoring devices. Thus, technologies are not only extensions of the nurse but they also mediate the relationship between the nurse and the patient shifting both the role and the responsibility of the patient and nurse in order to include the technology in the equation. In this vain we see that technologies already act to mediate all of the moral elements of the framework. In other words, we are not speaking of interactions that occur without the use of technologies. Therefore, the question is not what happens when a care robot enters the nurse-patient relationship that is devoid of any technologies. Rather, we are speaking of a context within which technologies are already employed to a high level and the question is how will a care robot alter the existing practice and further can the robot reintroduce elements that may have been overlooked from the previous round of automation?

\section{Type of Robot as a Component of the Framework}

The typifying of robots is done in many different ways. Some consider a type of robot according to the domain for which it is used; industrial versus rehabilitation 
versus military versus search and rescue robots (Veruggio and Operto 2006). For others, types of robots may be in terms of industrial robots versus service robots versus personal robots. This classification of robots is dependent on the amount of human interaction the robot will have and the predictability or structuring of the environment within which the robot is working. Industrial robots have very little interaction with humans and are present in a structured environment where their actions are closely monitored and highly predictable. Service robots are meant to act in human environments (unstructured and unpredictable) with varying degrees of human contact and interaction (Engelberger 1989). Personal robots are a type of service robot meant to interact and cooperate with humans in human environments - a domestic robot of sorts. There is no consensus as to a universal definition of a robot let alone the classification of different types of robots.

To specify for the purposes of the framework discussed here, the manner in which I classify as 'type of robot' has to do with how the robot will be used among the human actors - how a role and responsibility is delegated to a robot. For example, an enabling robot is one which enables a human to perform an action previously not possible without the robot or, the robot enhances the human's performance during a task - the robot and human are working together toward a goal but the human is in control of the both him/herself as well as the robot. Thus, the role and responsibility for accomplishing that role is a shared effort with the robot perceived in an instrumentalist way, as a tool. Robots of this type are telepresence robots like the RP7 or surgical robots like Intuitive Surgical's daVinci. A replacement robot is one that fulfills a practice in place of the human. The role of the human and the associated responsibilities are delegated fully to the robot. Examples of this type of robot are the RI-MAN or RIBA autonomous robots for lifting. An assistive robot is one which aids a human in performing an action by providing a portion of the practice without the direct input of a human operator and is thus delegated a partial role and a partial responsibility. This robot differs from an enabling robot in that it does not require consistent input from a human but rather can execute a practice once given its command. Examples of this kind of robot are the TUG and HelpMate robot used for deliveries in hospitals. The role and responsibility of the delivery is shared between the robot and the human deliverer/ receiver; however, the robot fulfills many steps without input from a human.

\section{Manifestation of Moral Elements as a Component of the Framework}

Manifestation of moral elements refers to how the values are observed, prioritized, and interpreted throughout a care practice, in a given context (with and without the introduction of the care robot). The values are expressed as the moral elements in care, identified by care ethicist Joan Tronto. They are attentiveness, responsibility, competence and reciprocity (Tronto 1993). These elements are general such that they may be considered needs of any care practice, independent of individual caregivers, care-receivers, context or practice. The moral elements act as a heuristic tool to ensure the incorporation and reflection of the fundamental care values in the design of a care robot. This component relies on a detailed description of the care practice such that the moral elements become apparent through the description of 
the practice, in context, in terms of the actions and interactions between actors (human and nonhuman). Along with understanding the way in which the moral elements come into being it is important to indicate the distribution of roles and responsibilities as an additional tool for observing the presence of norms in a given practice.

\section{The Care Centered Value Sensitive Design Methodology}

Applying the care centered framework allows me to analyze the components of good care practices with and without the presence of a care robot. When we operationalize the framework we are able to see the relationship between the technical content of a care robot and how it works to promote (or demote) certain care values. To follow the CCVSD methodology, one begins by identifying the context, practice, actors involved and how the moral elements are manifest in traditional care practices. This does not mean that the traditional care practice (i.e. the care practice without the care robot) provides a normative standard in all cases but rather to understand how values become manifest through the practice. Keeping in accordance with the care orientation, beginning in this way allows for an analysis with the emphasis on uncovering the distribution of responsibilities, roles and values.

As I have claimed, the framework may be used for both the retrospective and the prospective ethical assessment of care robots and the manner in which the CCVSD methodology occurs differs for each. In other words, it may be used at multiple times throughout the design process of a care robot. For retrospective evaluations using the framework, one identifies the context, practice, actors and the manifestation of moral elements for the practice without the inclusion of a care robot. Following this, one then discusses the type of robot (assistive vs. enabling vs. replacement) and the manner in which the proposed care robot capabilities impact the manifestation of moral elements. As such, the evaluation of the care robot is done on a design-by-design basis according to context and practice. For retrospective analysis, the CCVSD methodology allows one to evaluate the addition of the care robot into a network of actors performing a practice in a specific context. The methodology for retrospective evaluation incorporates additional reflective tools, in particular the theory of scripts (Akrich 1992), for uncovering the deeper meaning attributed to the robot through an analysis of the (potential) embedded assumptions as well as any shift in roles and responsibilities among actors.

For prospective evaluation, one again identifies the context, practice, actors and the manifestation of moral elements for the practice without the inclusion of a care robot. Following this, one then speculates on what capabilities a robot ought to have to ensure the promotion of said values. For prospective analysis, the CCVSD methodology allows engineers and ethicists to understand the capabilities the robot ought to have in order to safeguard the manifestation of care values. In order to demonstrate how the care centered framework and the CCVSD methodology may be used for the retrospective ethical evaluation of care robots, I take the practice of lifting and compare two care robots which may be used for this practice. 
Putting the Framework to Use for Retrospective Analysis: The Practice of Lifting

One of the more challenging practices for the nurse is the lifting of patients. Many elderly patients in the hospital or nursing home require partial assistance for lifting themselves out of bed or out of a chair. Alternatively, many are not capable of supporting their own weight at all and require complete assistance of a nurse to get out of bed or out of a chair. Given that the nurse must do this for any number of patients, there is a risk to the nurse's physical safety if she/he is required to lift every patient. What's more, many nurses are not physically strong enough for this. As a result, nurses have opted to use mechanical lifts on the many occasions that patients need to be lifted ( $\mathrm{Li}$ et al. 2004).

To do this the nurse encloses the curtain around the patient to ensure privacy throughout lifting. The nurse adjusts the bed for ease of lifting and using the mechanical lift for complete assistance, the patient is lifted using a remote control, controlled by the nurse. The patient is then lowered into the chair. When the patient is being lifted there is no physical contact with the nurse, although the nurse is physically present there is no chance for eye contact as the patient is raised quite high and the nurse is paying attention to the remote control and aligning the wheelchair for placement. Thus, eye contact and touch are not possible. This first wave of automation presents a rather flat view of the care practice of lifting. Meaning, it appears to have viewed the practice as a task, as an event that is separate from the process of care and uninvolved in the manifestation of care values. As I have already shown, valued actions like touch and eye contact are integral for establishing and/or maintaining a trusting bond and this bond is integral for the provision of good care later on in the process (the patient complying with their treatment plan, taking medications, being honest about their symptoms etc.). In short, the practice of lifting requires much more than the action of lifting the carereceiver from one place to another. In order to call this is a "good care practice" according to the care orientation, many other values need to be expressed throughout the practice-values like attentiveness to the care-receiver responses or eye contact for establishing trust in the practice (and technology in this instance).

For this example, the context is the hospital, the critical care ward, the practice is lifting and the actors are the care-receiver, the care-giver, the mechanical lift, the mechanical bed, the curtain to enclose the care-receiver and the room. In terms of the manifestation of moral elements, attentiveness of the nurse is directed towards the machine and its functioning rather than exclusively towards the patient and their status. In terms of responsibility, the nurse is responsible not only for their behavior but also for the function of the machine. Trust here is a hybrid affair between the care-giver, care-receiver and the mechanical lift-meaning, the patient trusts the nurse as to whether or not the mechanical lift will work. Trust is also bestowed on the technology as a result of the values guiding the institution-the patient trusts the institution's judgment when using the technology. In terms of competence, it is questionable whether the lift facilitates the element of competence. Although the nurse is able to lift multiple patients in a given day with the same skill, the lack of touch and eye contact leave one wondering whether or not this is a skillful 
completion of the task. The reason to question this once again has to do with the criteria for "good care"-fulfillment of the action efficiently is not enough to render a practice 'good', the manner in which the practice is fulfilled is what makes it a 'good' practice. Thus, a good lifting practice, in the hospital, includes eye contact as a way of communicating and establishing a bond. With respect to reciprocity, the nurse is present and thus verbal and visual communication are possible; however, with the nurse's attention on the remote one would wonder how perceptive they might be to the reactions of the patient. ${ }^{4}$ The current technology involved in the practice of lifting shows us how important it is for designers to understand the holistic vision of care and how care practices fit within this vision-they act as a moment for the promotion of care values. Consequently, the introduction of care robots presents a unique opportunity to re-introduce certain values of ethical importance. Alternatively, a robot may perpetuate the trend to minimize certain care values.

\section{Care Robots for Lifting and Their Impact on the Moral Elements of Care}

There are two robots which will be used for the CCVSD retrospective ethical evaluation of current care robot designs. Each of these robots is considered an actor in the practice of lifting once it is incorporated. The first is an autonomous robot for lifting, formerly known as the RI-MAN robot from the Riken Institute (Onishi et al. 2007) now replaced by the RIBA robot and the second is a human-operated exoskeleton, the Hybrid Assistive Limb (HAL) from Cyberdyne (Hayashi et al. 2005). There are a variety of exoskeletons currently on the market with similar capabilities and thus for the purposes of this analysis the important distinction to be made is between an autonomous and a human-operated robot. Both robots can achieve the same task (lifting a patient); however the technical capabilities through which this task is achieved differs and thus changes the way in which the caring practice is fulfilled. The autonomous robot meaning is capable of lifting a patient and carrying him/her from one place to another without being controlled by a human operator. This robot is a replacement robot, aimed to replace the care-giver in the practice of lifting. The robot is designed to work directly with humans and as such is programmed for safety considerations like speed as well as the materials which are used for its structure.

Alternatively, the human-operated robot, HAL, is an exoskeleton, meaning a human operator wears the robot in order for it to fulfill its task. The robot is a weight displacing robot such that the human does not feel the full effects of the weight. Versions of this type of robot exist in factory and military applications to prevent over exertion of factory workers or soldiers respectively. It is not an autonomous robot, but a human-operated one and is thus an enabling robot-one which does not replace a human in their role but shares the role with a human. It too will interact directly with a human (more than one in most instances) and must be programmed for the appropriate safety considerations. Given that the robot is human-operated,

\footnotetext{
${ }^{4}$ This is not a critique or nurses or the manner in which they fulfill their roles but rather a critique of the technology and its impact on the practice of lifting.
} 
programming for safety considerations are slightly different compared to those of the autonomous one. For example, the robot will not require the same sensors for perceiving a wall, person or object in its range. While the previous robot is capable of replacing the human care-giver that would normally lift the patient, this robot is meant to assist the human care-giver with their task. By reading the biometric signals of the care-giver, the robot is able to bear the burden of the weight of whatever the care-giver is lifting. This could be a patient, a bed, a heavy box etc.

\section{Manifestation of Moral Elements Once the Robot has been Introduced into the Care Practice}

The resulting practice of lifting when comparing the two robots looks incredibly different. In the first instance, the autonomous robot, all elements have been delegated to the robot. Meaning, the robot is responsible for being attentive to the frailty of the patient when lifting, the robot is ultimately responsible for the safety throughout the practice, the robot is required to fulfill the practice in a skillful manner and, the robot is responsible for perceiving whether the needs of the patient have been met. Firstly, at this point in time, the technology does not allow for such a sophisticated manner of task completion by a robot-the robot does not know what it is doing or why it is doing something and is not capable of engaging in a reciprocal interaction. The question then is whether the robot's capabilities ought to be evaluated against the human or whether a new standard ought to exist for robots. From a moral perspective, given that the robot is a replacement robot in a nursing home context, the robot must be evaluated against a human care-giver. The same does not hold for an enhancement or assistive robot. From Tronto's perspective, which asserts that care is only good care when there is a marriage between caring about and caring for, the robot does not meet the requirements of a good carer. From Little's position of the care orientation, emphasis is drawn to the care robot's possibility to interfere with the establishment of trust and bonds and in so doing the care robot poses a threat. As such, the moral question is not whether or not the robot can fulfill the practice in an efficient manner but why the robot is being delegated this role and responsibility. Alternatively, from a technical perspective this does not demand that the robot's appearance mimic a humans - the autonomous robot could perhaps have four arms instead of two to facilitate lifting. But, from a moral stance, a robot replacing a human must be assessed according to whether or not it is capable of facilitating and promoting the vales a human care-giver brings about.

If, however, the robot is (someday) capable of understanding what it is doing and why, and may act in a skillful manner, the robot still poses a threat to the holistic process of care. The holistic process of care refers to the concept that care is not one task or a series of tasks but is a compilation of practices to meet the needs of the actors, each practice building on the last. For the practice of lifting it is necessary that the care-receiver trust the care-giver initially in order for lifting to occur but it is also a moment in which the two can establish a bond to ensure compliance with a care plan further along. Seeing as the robot replaces the care-giver for this practice, it is possible that the care-giver and care-receiver may not have the same bond-the care-receiver may not feel any sort of responsibility towards the care-giver to abide 
by their care plan. What's more, perhaps the care-receiver may not trust the plan established by the care-giver. This is not to say that trust cannot be established through another practice, but rather that it does not present the forum in which trust is traditionally established or strengthened. Taking this into consideration in the design process of the robot means that designers ought to anticipate this and perhaps consider another forum in which trust can be established or strengthened between the care-giver and care-receiver.

Alternatively, in another context there may be care-receiver's who would prefer the assistance of an impartial robot to keep their dignity and integrity intact. This could be the case in a home setting where a spouse is the only one available for lifting. With a change in context comes a change in the interpretation and prioritization of values. The care-giver may be the spouse of the care-receiver and thus privacy is seen in a different way. Instead of the curtain being closed as a way to ensure privacy, the care-receiver would prefer not to be exposed in that way to their spouse at all. This interpretation of the value of privacy may place it at the top of the hierarchy of values above others like efficiency, eye contact, touch or human presence. Consequently, having an autonomous robot to fulfill the practice of lifting may be seen as a more compassionate means when the care-receiver's vulnerability is maximized by requiring help for these practices. Once again, this divergence shows the importance of context in the ethical assessment of a care robot.

In the second instance, the case of the enabling robot, the element of attentiveness is still in the domain of the human as is the element of responsiveness. For the former, the care-giver uses his/her own faculties to ascertain when the carereceiver needs to be lifted, at what speed, from which angle and with or without social interaction. For the latter, reciprocity is something that happens between the care-giver and care-receiver in real time by verbal and nonverbal cues detected by the care-giver. Meaning, the nurse can ask the patient how they are doing while they are lifting. As for responsibility and competence, these elements now become shared endeavors between the human and the robot given that the role of weightbearer is delegated to the robot. The care-receiver and care-giver must both trust the technology-responsibility for the safety of the practice becomes a hybrid event between the human care-giver and the robot. Additionally, a certain amount of competence for the skillful completion of the practice is delegated to the robot. Thus, a portion of the responsibility for lifting is delegated to the robot as is a certain level of skill; however, this is done in an enabling way, therefore the human care-giver is still responsible overall. This also means that the robot is not evaluated against the human completely but is evaluated as to how it enables the human in their performance of the practice. As such the robot is evaluated according to how well it bears the weight but not how well is it able to pick up on nonverbal cues of the care-receiver.

The reflections provided here give preliminary insights into the impact of a care robot and its capabilities on the resulting care practice. This is not an exhaustive reflection, however, given that a deeper meaning may be attributed to the inscribed shift in roles and responsibilities. Accordingly, the CCVSD methodology incorporates additional tools for an in-depth look at the meaning the robot may take on. 


\section{Attributing Meaning to Care Robot Designs}

It is only through a deeper understanding of what care values are and how they are manifest throughout a care practice that we come to grasp the impact a design might have on the care practice. Above and beyond the direct relationship one might uncover between care values and the technical capabilities of the care robot, there is greater meaning attributed to these capabilities upon further reflection.

Akrich discusses the embedding of elements in terms of assumptions made about user preferences and competencies (1992). Placed in context, each robot takes on a distinctive meaning related to the assumptions embedded within. This description is quite useful for my reflection and an important distinction must be made here pertaining to the difference between assumptions and the concept of values and norms. Assumptions are more about the real word, they are descriptive in a sense while values are more about what the real world ought to be like, they are normative in a sense. When an assumption is made about a value to be embedded, it does not have to be a description about what is, but could also be a claim about what values ought to be expressed, how they ought to be expressed, or what priority they ought to be given. In others words, when the built-in assumption pertains to a value, or when a valuation is being made, the result is a normative claim about what the values should be, what should be valued, or what the ideal is. For Akrich, "many of the choices made by designers can be seen as decisions about what should be delegated to a machine and what should be left to the initiative of human actors" ( $p$. 216). By making choices about what should and should not be delegated to certain actors (human or nonhuman), engineers may change the distribution of responsibilities in a network.

Consequently, each robot reflects divergent assumptions pertaining to the understanding of a care practice, the aim of the care practice and the prioritization of values manifest through a care practice. When using the autonomous robot in a hospital setting, the understanding of the practice reflects a vision of a task rather than a practice-that lifting is just an action that needs to be done in order to get on to the next action. As I have stressed throughout the paper, from the care ethics perspective this is not what a good care practice looks like. The ideal practice of lifting seen through this robot is a standardized one where the value of efficiency is placed as the top priority. Although efficiency was not explicitly discussed previously, it is thought to fall under the realm of competence. This onedimensional view of good care as efficient may have negative implications for the overall care process. One may presume that the quality of interactions, the number of social interactions, and the presence of a human are threatened by this efficient system. Alternatively, the system may be considered efficient given that time of the human care-giver is freed up, ultimately improving the number of social interactions and the quality thereof. This last point is also dependent on how the robot is introduced and would not become apparent until the robot has been implemented in a specific context.

The autonomous robot reflects a vision of the practice of lifting which does not require any of the values traditionally involved; human touch, eye contact, human presence. If these values are normatively understood and recognized as only 
possible through human-human interactions, ${ }^{5}$ then this demands that a human be present for the practice of lifting in all instances. However, as we saw before, context plays a role. It is possible to suggest that in the context of the hospital or nursing home, where "good care" depends on the relationship between the caregiver and care-receiver, human contact for a practice like lifting is always required. This is in part due to the vulnerability of the care-receiver while being lifted as well as the need to form a bond between care-giver and care-receiver. Alternatively, in a home context in which a relationship between care-giver and care-receiver is already established and strengthened, the need for human presence, eye contact and touch for the practice of lifting may not be as pertinent. Moreover, when the caregiver is a spouse it may be preferable not to have the human present. Thus, both design and integration into the healthcare system are of importance here.

Alternatively, the human-operated exoskeleton reflects an understanding of this care practice as one in which the aim of the practice is not solely to lift the care-receiver from one place to another but is a moment to establish a bond and convey other care values. The vision of care presupposed in the design of the human-operated care robot is one in which individualized care with a human care-giver present at all times for all parts of the care practice, is the overall aim. Efficiency is still a priority; however, it is achieved through meeting the need of the care-giver by contributing to the element of competence (enhancing the skill with which the care-giver may perform their role), attentiveness, (enabling the care-giver to perceive the minute cues of the care-receiver through the practice of lifting), and responsiveness (closely aligned with attentiveness but also embodies the reciprocal dimension of the relationship). Consequently, by demanding the human's presence for the task of lifting, the robot pays tribute to the holistic vision of care and the intertwining of needs and values.

I cannot say whether this is the epistemic aim of engineers, but can only point to the potential meaning that the robot may take on through pervasive use, and the presupposing assumptions directing such a meaning. Moreover, this is not to say that the autonomous robot ought to be disregarded or labeled as unethical-a variation in context changes things. Clearly, decisions concerning the use of a robot and its ethical implications are many-sided and complicated and demand an understanding of the specific context and users for anticipating how the elements will be served to their greatest potential.

\section{Conclusion}

The prospective robots in healthcare intended to be included within the conclave of the nurse-patient relationship require rigorous ethical reflection to ensure that their design and introduction do not impede the promotion of values and the dignity of patients at such a vulnerable and sensitive time in their lives. The ethical evaluation of care robots requires insight into the values at stake in the healthcare tradition. What's more, given the stage of their development and the lack of standards to

\footnotetext{
$\overline{5}$ I say 'many' of these values given that certain telepresence robots are capable of providing eye contact and the feeling or impression of human presence even when the human is not physically present.
} 
guide their development, ethics ought to be included within the design process of such robots. The manner in which this may be accomplished, as presented here, uses the blueprint of the Value-sensitive design approach as a means for creating a framework tailored to care contexts. Using care values as the foundational values to be integrated into a technology and using the elements in care as the normative criteria, the resulting approach is referred to here as "care centered value-sensitive design".

The care centered framework is meant to indicate and direct the evaluator to the necessary components in care from a care orientation. The CCVSD methodology is meant to provide a guideline for analysis of a practice with and without the use of a care robot. Using the CCVSD methodology to compare two care robots used for the same practice with different capabilities, allows us to envision the resulting care practice in terms of the robot's impact on care values as well as the robot's potential impact on care in the holistic sense. For the latter, this is only understood when one grasps the interconnectedness of one practice with another. From this, the link between robot capabilities and their impact on the manifestation of care values is made clear. Consequently, we may ask the question; what kind of care do we want to provide and in so doing we may steer the design and development of care robots.

The aim of this paper was to present the conceptual foundation for the creation of a framework and methodology for the evaluation of care robots both retrospectively and prospectively. The aim was not to present an exhaustive evaluation of current and future care robots as this will be taken up in a later paper. The significance of this work comes from the stage of development of care robots and the belief that ethics may be included at this time in the design process to foster trust between the public and the resulting robots. The care centered framework adheres to the central thesis of the care orientation - that one is oriented to the components which require attention in order to begin ethical deliberation. What's more, the framework provides a starting point for the interdisciplinary collaboration of a range of robotics researchers-from designers, engineers and computer programmers to ethicists, psychologists and philosophers.

Open Access This article is distributed under the terms of the Creative Commons Attribution Noncommercial License which permits any noncommercial use, distribution, and reproduction in any medium, provided the original author(s) and source are credited.

\section{References}

Akrich, M. (1992). The de-scription of technical objects. In W. Bijker \& J. Law (Eds.), Shaping technology/building society. Cambridge, MA: MIT Press.

Asaro, P. (2006). What should we want from a robot ethic? In R. Capurro \& M. Nagenborg (Eds.), Ethics and robotics. Amsterdam, The Netherlands: IOS Press.

Barras, C. (2009). Useful, loveable and unbelievably annoying. The New Scientist, 22-23.

Brey, P. (2009). Values in technology and disclosive computer ethics. In L. Floridi (Ed.), The Cambridge handbook of information and computer ethics. Cambridge: Cambridge University Press.

Cummings, M. (2006). Integrating ethics through value sensitive design. Science and Engineering Ethics, 12(4), 701-715.

Engelberger, J. (1989). Robotics in service. London: MIT Press. 
Freidman, B., Kahn, P., et al. (2006). Value sensitive design and information systems. In P. Zhang \& D. Galletta (Eds.), Human-computer interaction in management information systems: Foundations (pp. 348-372). NY: M. E. Sharpe.

Friedman, B., \& Kahn, P. H., Jr. (2003). Human values, ethics, and design. In The human-computer interaction handbook: Fundamentals, evolving technologies and emerging applications (pp. 1177-1201). Mahwah, NJ: Lawrence Erlbaum Associates, Inc.

Gadow, S. A. (1985). Nurse and patient: The caring relationship. In A. H. Bishop \& J. R. Scudder Jr. (Eds.), Caring, curing, coping: Nurse, physician, patient relationships (pp. 31-43). University Alabama: The University of Alabama Press.

Gilligan, C. (1982). In a different voice: Psychological theory and women's development. Cambridge, MA: Harvard University Press.

Hayashi, T., Kawamoto, H., \& Sankai, Y. (2005). Control method of robot suit HAL working as operator's muscle using biological and dynamical information. IEEE International Conference on Intelligent Robots and Systems, 3063-3068.

Howcroft, D., Mitev, N., \& Wilson, M. (2004). What we may learn from the social shaping of technology approach. In J. Mingers \& L. P. Willcocks (Eds.), Social theory and philosophy for information systems (pp. 329-371). Chichester: Wiley.

ISO. (2011). http://www.iso.org/iso/iso_catalogue/catalogue_tc/catalogue_detail.htm?csnumber=53820.

Jecker, N. S., Carrese, J. A., \& Pearlman, R. A. (2002). Separating care and cure: An analysis of historical and contemporary images of nursing and medicine. In E. Boetzkes \& W. J. Waluchow (Eds.), Readings in healthcare ethics (pp. 57-68). Canada: Broadview Press.

Kawamoto, H., \& Sankai, Y. (2002). Power assist system HAL-3 for gait disorder person. Computers Helping People with Special Needs; Lecture Notes in Computer Science, 2398, 19-29.

Latour, B. (1992). Where are the missing masses? The sociology of a few mundane artifacts. In W. Bijker \& J. Law (Eds.), Shaping technology/building society. Cambridge, MA: MIT Press.

Le Dantec, C., Poole, E., \& Wyche, S. (2009). Values as lived experience; evolving value sensitive design in support of value discovery. In Proceedings of the 27th International conference on human factors in computing systems. ISBN: 978-1-60558-246-7.

Li, J., Wolf, L., \& Evanoff, B. (2004). Use of mechanical patient lifts decreased musculoskeletal symptoms and injuries among health care workers. Injury Prevention, 10(4), 212-216.

Little, M. (1998). Care: From theory to orientation and back. Journal of Medicine and Philosophy, 23(2), 190-209.

Mander-Huits, N. (2011). What values in design? The challenge of incorporating moral values in design. Science and Engineering Ethics, 17, 271-287.

Metzler, T., \& Lewis, L. (2008). Ethical view, religious views, and acceptance of robotic applications: A pilot study. Association for the Advancement of Artificial Intelligence. http://www.aaai.org.

Nathan, L., Friedman, B., Klasnja, P., Kane, S., \& Miller, J. (2008). Envisioning systemic effects on persons and society throughout interactive system design. In Proceedings of DIS (pp. 1-10).

Nissenbaum, H. (1998). Values in the design of computer systems. Computers and Society, 38-39.

Noddings, N. (1984). Caring: A feminine approach to ethics and moral education. Berkely: University of California Press.

Onishi, M., ZhiWei, L., Odashima, T., Hirano, S., Tahara, K., Mukai, T. (2007). Generation of human care behaviours by human-interactive robot RI-MAN. IEEE International Conference on Robotics and Automation, 3128-3129.

Reich, W. T. (1995). History of the notion of care. In W. T. Reich (Ed.), Encyclopedia of Bioethics, vol. 5 (pp. 319-331), Revised edition. New York: Simon \& Schuster Macmillan.

Rosati, C. (2009). Relational good and the multiplicity problem. Philosophical Issues, 19(1), $205-234$. Ruddick, S. (1995). Maternal thinking. New York: Ballantine Books.

Sharkey, N., \& Sharkey, A. (2010). Granny and the robots: Ethical issues in robot care for the elderly. Ethics and Information Technology. doi:10.1007/s10676-010-9234-6.

Simpson, J. A., \& Weiner, E. S. C. (1989). The Oxford english dictionary. Oxford: Clarendon Press.

Sparrow, R., \& Sparrow, L. (2006). In the hands of machines? The future of aged care. Mind and Machine, 16, 141-161.

Super, D. E. (1973). The work values inventory. In D. G. Zytowski (Ed.), Contemporary approaches to interest measurement. Minneapolis: University of Minnesota Press.

Tronto, J. (1993). Moral boundaries: A political argument for an ethic of care. New York: Routledge.

Tronto, J. C. (2010). Creating caring institutions: Politics, plurality, and purpose. Ethics and Social Welfare, 4(2), 158-171. 
Vallor, S. (2011). Carebots and caregivers: Sustaining the ethical ideal of care in the 21st century. Journal of Philosophy and Technology, 24, 251-268.

Van den Hoven, J. (2007). ICT and value sensitive design. International Federation for Information Processing, 233, 67-72.

Van Wynsberghe, A., \& Gastmans, C. (2008). Telesurgery: An ethical appraisal. Journal of Medical Ethics, 34, e22.

Vanlaere, L., \& Gastmans, C. (2011). A personalist approach to care ethics. Nursing Ethics, 18(2), 161-173.

Verbeek, P. (2006). Materializing morality: Design ethics and technological mediation. Science, Technology and Human Values, 31(3), 361-380.

Verbeek, P. (2008). Morality in design; design ethics and the morality of technological artifacts. In P. Vermaas, P. Kroes, A. Light, \& S. Moore (Eds.), Philosophy and design: From engineering to architecture (pp. 91-102). Berlin: Springer.

Veruggio, G., \& Operto, F. (2006). Roboethics: A bottom-up interdisciplinary discourse in the field of applied ethics in robotics. In International Review of Information Ethics. Ed. Ethics in Robotics, pp. $2-8$.

WHO. (2010). Health topics: Ageing. Available from: http://www.who.int/topics/ageing/en/.

Wilson, M. (2002). Making nursing visible? Gender, technology and the care plan as script. Information Technology and People, 15(2), 139-158.

World Health Organization. (2007). People centered health; a framework for policy. http://www.wpro. who.int/NR/rdonlyres/55CBA47E-9B93-4EFB-A64E-21667D95D30E/0/PEOPLECENTRED HEATLHCAREPolicyFramework.pdf. 